\title{
AN APPROPRIATE INFLOW MODEL FOR SIMULTANEOUS DISSOLUTION AND DEGRADATION
}

\author{
Ju-Hyun Lee, Sungkwon Kang* and Hoo-Kyun Choi
}

\begin{abstract}
Based on the observed data for Clarithromycin released, three commonly used inflow models: the power, the exponential, and the logarithmic models are considered. Among them, the power model is used most in practice for simplicity. Using the numerical parameter estimation techniques, the parameters appeared in the model equations are estimated. Through the numerical estimation results using the several experimental data sets, the exponential model turns out to be best among the three models. More specifically, the sum of squares of absolute errors and the sum of squares of relative errors for the exponential model are reduced by $80-95$ $\%$ for the experimental data sets and $60-90 \%$ for the noise added data sets compared with those for the power and logarithmic models. A typical experimental data set is used in this paper to show the estimation method and its numerical results. The proposed numerical method and its algorithm are designed for estimating the parameters appeared in the model differential equations for which the exact form of the solution is unknown in general. The methodology developed can be applied to more general cases such as the nonlinear ordinary differential equations or the partial differential equations.
\end{abstract}

\section{Introduction}

Recently, considerable attentions have been focused on the dissolution test of oral dosage forms. It plays an important role both in the development of a new drug product and the quality control of a marketed product $[7,10,16,20]$. The solubility, the dissolution rate, and the intestinal

Submitted January 23, 2009. Accepted March 9, 2009.

2000 Mathematics Subject Classification. 65M06, 65M32, 65Y20

Key words and phrases. Clarithromycin, dissolution, degradation, differential equation, parameter estimation

*Corresponding author. The research of this author was supported by Chosun University Research Funds 2007. 
permeability have been considered as the major biopharmaceutic factors that determine the extent and the rate of absorption[15]. The dissolution test becomes more important when a drug is classified as Class II or Class IV in BCS(Biopharmaceutics Classification System) $[8,15]$, since the extent of absorption can be limited by the dissolution rate of the drug. In addition, the dissolution rate of a drug can be correlated with the in vivo results when an appropriate test method is used for the dissolution study. Therefore, it is important to measure the dissolution rate of a drug product accurately using appropriate method for the product. However, when a drug is degraded during dissolution study, the extent of dissolution can be erroneously estimated[10]. Depending on analytical method used, it can be underestimated or overestimated. It has been reported in the literature that some of the drugs could be degraded during the dissolution study $[9,11,12,14,16,21]$. It is essential to know the amount of drug released and the amount of drug degraded during the dissolution study in the development of suitable formulation for the degrading drug and subsequent quality control of the developed product. If all the degradation products can be quantitatively analyzed, it will be easy to measure both the amount of drug released and the amount degraded. However, it is very difficult to measure the amount of degradation product accurately. Several mathematical approaches have been made to describe simultaneous dissolution and degradation[7,21].

In this study, we develop a mathematical model to estimate the amount of drug released based on the amount of drug left after degradation in the dissolution medium. From the observed data of the model drug, Clarithromycin, an appropriate inflow model is found. Three typical inflow models: the power, the exponential, and the logarithmic models are considered. Among the models, the power model is used most in practice for simplicity. Since the inflow models are given as parts of the corresponding governing differential equations describing the degradation, and the exact forms of the solutions for the equations are unknown in general, the parameters appeared in each model are estimated based on the observed data and the numerical estimation techniques. For the parameter estimation techniques, see [1,3-6,17] and the references therein. For the error analysis, the sum of squares of absolute errors(SSA) and the sum of squares of relative errors(SSR) are considered. From the numerical estimation results, the exponential inflow model turns out to be best among the three proposed models. More specifically, the SSA and the SSR for the exponential inflow model are 
An Appropriate Inflow Model for Simultaneous Dissolution and Degradation 111

reduced by approximately 80-95\%, compared with those for the power and the logarithmic models. Also, by allowing the maximum of $\pm 2 \%$ random noise on the observation data, the reduction rates of the SSA and the SSR for the exponential model compared to other two models are ranged from $60 \%$ to $90 \%$.

In Section 2, the materials and the methods for obtaining the experimental data are explained. The mathematical models and the numerical parameter estimation schemes are described in Section 3. The numerical estimation results using a typical experimental data set are given in Section 4 .

\section{Materials and methods}

\subsection{Materials}

Clarithromycin was a gift from Chong Kun Dang Pharmaceutical Company (Seoul, Korea). All other chemicals were of reagent grade or above and were used without further purification.

\subsection{Release of Clarithromycin from the tablet}

Drug release test was carried out using a dissolution tester(DST 810, Labfine, Inc., Korea). Clarithromycin tablets were placed in $900 \mathrm{ml}$ of the dissolution medium at $37^{\circ} \mathrm{C}$ using USP dissolution apparatus II(paddle method) with a paddle rotating at $50 \mathrm{rpm}$. The $\mathrm{pH}$ of dissolution mediums tested was 1.2. An aliquot of release medium was withdrawn at predetermined time intervals and equivalent amount of fresh medium was added. Withdrawn samples were passed through a $0.45 \mu \mathrm{m}$ nylon filter and immediately neutralized with $0.1 \mathrm{~N} \mathrm{NaOH}$ solution and analyzed using HPLC(Shimadzu Scientific Instruments, MD, USA) to determine the amount of Clarithromycin released from the tablet.

\subsection{Stability of Clarithromycin in $\mathrm{pH} 1.2$ buffer}

Stability test was carried out using a dissolution tester(DST 810, Labfine, Inc., Korea). Clarithromycin $(50 \mu \mathrm{g} / \mathrm{ml})$ was dissolved in $\mathrm{pH}$ 1.2 buffer preheated to $37^{\circ} \mathrm{C}$. An aliquot of the buffer was withdrawn at predetermined time points. The withdrawn samples were immediately 
neutralized with $0.1 \mathrm{~N} \mathrm{NaOH}$ to prevent further degradation. The concentration of Clarithromycin was measured by HPLC system(Shimadzu Scientific Instruments, MD) at the wavelength of $210 \mathrm{~nm}$.

\section{Mathematical models and parameter estimation}

\subsection{Mathematical models}

To describe the amount of intact drug left in the dissolution medium, the following differential equation are considered.

$$
\begin{aligned}
\frac{d}{d t} Q_{m e}(t) & =\frac{d}{d t} Q_{i n}(t)-k Q_{m e}(t), \quad t>0, \\
Q_{m e}(0) & =0,
\end{aligned}
$$

where $Q_{m e}(t)$ is the amount of the intact drug remaining in the medium(\%) measured at time $t, Q_{\text {in }}(t)$ is the inflow quantity(the total amount of the drug released, \%) at time $t$, and $k$ is the first order degradation rate constant.

To find an appropriate inflow model, we consider the following three models.

Model 1. Power model: $Q_{\text {in }}(t)=a t^{n}$, where $a$ and $n$ are unknown.

Model 2. Exponential model: $Q_{i n}(t)=a\left(1-e^{-b t}\right)+c\left(1-e^{-d t}\right)$, where $a, b, c$, and $d$ are unknown.

Model 3. Logarithmic model: $Q_{i n}(t)=y_{0}+a \ln (t+b)$, where $a$ and $b$ are unknown, and $y_{0}=-a \ln b$ due to $Q_{\text {in }}(0)=0$.

Then, by plugging $Q_{\text {in }}(t)$ into equation (1), we have the following governing equations for each model.

$$
\frac{d Q_{m e}(t)}{d t}=a n t^{n-1}-k Q_{m e}(t), \quad Q_{m e}(0)=0
$$

for the power model,

$$
\frac{d Q_{m e}(t)}{d t}=a b e^{-b t}+c d e^{-d t}-k Q_{m e}(t), \quad Q_{m e}(0)=0
$$

for the exponential model, and

$$
\frac{d Q_{m e}(t)}{d t}=\frac{a}{t+b}-k Q_{m e}(t), \quad Q_{m e}(0)=0
$$

for the logarithmic model. 
Based on the measurement data of Clarithromycin released, we need to determine which model is best among the above three models. For this purpose, we assume that the governing equations (3)-(5) represent the amount(\%) of Clarithromycin remained in the medium. By finding the "optimal" parameters appeared in each model fitting the observed data "best", we can estimate the amounts $Q_{m e}(t)$ of the Clarithromycin remained at the times when the actual measurements are performed. Therefore, by comparing the estimated amounts in each model to the actual measurement ones, we can determine which is the most appropriate model among the proposed models. In these comparison, we also need to take account of measurement random noise errors. Therefore, the essential factor is to find the optimal parameters for each model under a certain accuracy tolerance level. In the following, we consider the general procedure of the parameter estimation for the problems described by the differential equations (3)-(5).

\subsection{Parameter estimation}

We begin with the actual measurement data set. Let

$$
w_{i}, \quad i=1,2, \cdots, m
$$

be the observed Clarithromycin remained data(\%) measured at time $t_{i}$. In each model (3), (4), or (5), the numerically or analytically estimated amounts $Q_{m e}\left(t_{i}\right), i=1,2, \cdots, m$, represent the measured data $w_{i}$. Let

$$
q=[a, n]^{T} \quad\left(q=[a, b, c, d]^{T} \text { for (4) and } q=[a, b]^{T} \text { for }(5)\right)
$$

be the parameters vector appeared in (3) ((4) or (5), respectively), where $T$ stands for the transpose, i.e., $q$ is a column vector. Then we consider the following parameter estimation problem:

Problem 1: Given the set of Clarithromycin measurements $w_{i}$ in (6), find the optimal parameter vector $q^{*}$ that minimizes the cost functional

$$
J(q)=\sum_{i=1}^{m}\left\{Q_{m e}\left(t_{i} ; q\right)-w_{i}\right\}^{2},
$$

where $Q_{m e}\left(t_{i} ; q\right)$ is the estimated solution at time $t_{i}$ for equation (3) ((4) or (5)) with the parameter vector $q$ defined by (7).

The general procedure for solving Problem 1 is following.

Step 1. Choose an initial guess vector $q:=q_{0}$. 
Step 2. From the current $q$, find a new $q_{\text {new }}$ such that $0 \leq$ $J\left(q_{\text {new }}\right)<J(q)$, and set $q:=q_{\text {new }}$. Here, $J$ is defined by (8).

Step 3. Until $J(q)$ reaches the minimum, continue Step 2.

Step 2 is the key step in the optimization process. First of all, for a given parameter vector $q$, we need to compute $J(q)$. As we see in (8), the computation of $J(q)$ requires the estimated values $Q_{m e}\left(t_{i} ; q\right)$ for times $t_{i}, i=1,2, \cdots, m$. However, $Q_{m e}(t ; q)$ is the solution of the differential equation $(3)((4)$ or $(5))$, and the exact form for the solution is unknown in general. Therefore, we need to employ an appropriate numerical method for solving the differential equations. Note that, from the theory of ordinary differential equations, equation (4) has the exact solution given by

$$
Q_{m e}(t)=\frac{a b}{k-b}\left(e^{-b t}-e^{-k t}\right)+\frac{c d}{k-d}\left(e^{-d t}-e^{-k t}\right) .
$$

The purpose of this paper is to provide a general parameter estimation method for solving the problems for which the exact solution forms are unknown as in the cases (3) and (5). Thus, we will consider the efficient and accurate numerical integration methods to which all the cases (3)(5) can be applied.

Another important issue in Step 2 is to find a new parameter vector $q_{\text {new }}$ from the current one $q_{\text {cur }}$ satisfying $J\left(q_{\text {new }}\right)<J\left(q_{\text {cur }}\right)$. To find a descent direction for $J(q)$, we need the information on $\nabla_{q} J(q)$, the gradient of $J$ with respect to the parameter $q$, or equivalently, $\nabla_{q} Q(t ; q)$, the gradient of $Q(t ; q)$ with respect to $q$. Since $Q(t ; q)$ is not available in an exact form as mentioned before, we need a numerical scheme for approximating $\nabla_{q} Q(t ; q)$. In this step, several time integrations will be needed. Also, a robust optimization method is needed due to the highly nonlinear dependency of $Q(t ; q)$ on $q$. Also, note that, at each iteration for updating $q$, the computations for $\nabla_{q} Q(t ; q)$, and, hence, many time integrations for solving the differential equations $(3)((4)$ or (5)) are required.

The stopping criterion for Step 3 can be given by $\left|J\left(q_{\text {new }}\right)-J\left(q_{\text {cur }}\right)\right|<$ tol for an appropriate accuracy tolerance, equivalently, by $\left|q_{\text {new }}-q_{\text {cur }}\right|<$ tol for many cases. From the brief explanation for Steps 2-3, in essential, many numerical integration methods for the differential equations 
$(3)\left((4)\right.$ or (5)) are required to obtain the optimal parameter $q^{*}$ minimizing the cost functional $J(q)$. Thus, the parameter estimation method should fulfill the following conditions:

(i) The time integration solver for the differential equations should be efficient and accurate.

(ii) The formulation for computing $\nabla_{q} Q(t ; q)$ should be simple, but, should produce accurate approximation.

(iii) The descent direction search algorithm should be robust.

Considering those aspects mentioned above, we will derive our parameter estimation algorithm.

3.2.1. Time integration. We now consider the numerical integration methods for solving the differential equations (3)-(5). There are many integration methods such as the Runge-Kutta method, the Crank-Nicolson method, multi-step methods, semi-implicit methods, etc.[2,19,22]. Since the equations are linear and relatively simple, the 4-th order RungeKutta method is employed in this paper to maintain the stability, convergency, accuracy, and efficiency. The total computational costs are within a few seconds.

The basic idea of the 4-th order Runge-Kutta method for solving the differential equation

$$
\frac{d}{d t} Q(t ; q)=f(t, Q(t ; q))
$$

can be approximated by

$$
Q(t+\Delta t ; q)=Q(t ; q)+\frac{\Delta t}{6}\left\{K_{1}+2 K_{2}+2 K_{3}+K_{4}\right\}
$$

where $Q(t ; q)=Q_{m e}(t ; q), f(t, Q(t ; q))$ represents the right hand side of equations (3), (4), or (5), $\Delta t$ is the time step for integration, and

$$
\begin{aligned}
& K_{1}=f(t, Q(t ; q)), \\
& K_{2}=f\left(t+\frac{\Delta t}{2}, Q(t ; q)+\frac{\Delta t}{2} K_{1}\right), \\
& K_{3}=f\left(t+\frac{\Delta t}{2}, Q(t ; q)+\frac{\Delta t}{2} K_{2}\right), \\
& K_{4}=f\left(t+\Delta t, Q(t ; q)+\Delta t K_{3}\right),
\end{aligned}
$$

and the parameter vector $q$ is given by $q=[a, n]^{T}, q=[a, b, c, d]^{T}$, and $q=[a, b]^{T}$ for equations (3), (4), and (5), respectively. From equation $(11)$, the estimated Clarithromycin amounts $Q\left(t_{i} ; q\right)$, and, hence, $J(q)$ 
in (8) can be obtained for each given time $t_{i}$ and the parameter vector $q$. From now on, $Q_{m e}(t)$ will be denoted by $Q(t)$ for notational purpose.

3.2.2. Calculation of $\nabla_{q} Q(t ; q)$. This step is for computing $\nabla_{q} J(q)$, the gradient of the cost function $J$ in (8), which is needed for finding a new parameter $q_{\text {next }}$ from the current one $q_{\text {cur }}$. Since this process involves the time integration for $Q(t ; q)$, it would be better to reduce the number of integrations, because the time integrations, i.e., solving the differential equations, are main sources for the computational costs required by the optimization process. Conventionally, Euler type method is used for the calculation of $\nabla_{q} Q(t ; q)$. However, we use the central difference method to obtain the accuracy, efficiency, and stability for our approximation scheme. Let $q$ be a parameter vector given by

$$
q=\left[q_{1}, q_{2}, \cdots, q_{l}\right]^{T},
$$

where $q_{i}$ is the parameters to be estimated for each model and $l$ is the number of parameters. That is, $l=2$ for the power and the logarithmic models and $l=4$ for the exponential model, and $a=q_{1}, n=q_{2}, a=$ $q_{1}, b=q_{2}, c=q_{3}, d=q_{4}$, and $a=q_{1}, b=q_{2}$ in (11) for each model. Then we have the following approximation for the gradient of $Q(t ; q)$ with respect to the parameter vector $q$.

$$
\nabla_{q} Q(t ; q)=\left[\frac{\partial Q(t ; q)}{\partial q_{1}}, \frac{\partial Q(t ; q)}{\partial q_{2}}, \cdots, \frac{\partial Q(t ; q)}{\partial q_{l}}\right]^{T},
$$

where, for each $i=1,2, \cdots, l, \frac{\partial Q(t ; q)}{\partial q_{i}}$ is approximated by the central difference:

$$
\begin{aligned}
\frac{\partial Q(t ; q)}{\partial q_{i}} & =\frac{Q\left(t ; \hat{q}_{i}^{+}\right)-Q\left(t ; \hat{q}_{i}^{-}\right)}{2 \Delta q_{i}}, \\
Q\left(t ; \hat{q}_{i}^{+}\right) & =Q\left(t ; q_{1}, \cdots, q_{i-1}, q_{i}+\Delta q_{i}, q_{i+1}, \cdots, q_{l}\right), \\
Q\left(t ; \hat{q}_{i}^{-}\right) & =Q\left(t ; q_{1}, \cdots, q_{i-1}, q_{i}-\Delta q_{i}, q_{i+1}, \cdots, q_{l}\right),
\end{aligned}
$$

where $\Delta q_{i}$ is a sufficiently small positive number. Note that two time integrations for $Q(t ; q)$ are needed for each time $t$ to obtain (18) for each $i=1,2, \cdots, l$. Since we need the information on $\frac{\partial Q\left(t_{j} ; q\right)}{\partial q_{i}}$ for each time $t_{j}$, $j=1,2, \cdots, m$, by saving the previous computational results $\frac{\partial Q\left(t_{j-1} ; q\right)}{\partial q_{i}}$, the actual computational costs are similar to those for the Euler type method. In order words, by the approximation (18), we can obtain the high accuracy with almost minimum computational costs. Also, this approximation is stable by using the balanced information $Q\left(t ; \hat{q}_{i}^{+}\right)$and $Q\left(t ; \hat{q}_{i}^{-}\right)$. These are the strong points of employing the central difference 
method for approximating the gradient of $Q(t ; q)$ with respect to each component $q_{i}$.

3.2.3. Descent direction and algorithm. We now consider the problem for finding a new parameter vector $q_{\text {next }}$ from the current one $q_{\text {cur }}$ satisfying the condition $0 \leq J\left(q_{\text {next }}\right)<J\left(q_{\text {cur }}\right)$. Updating the parameter vector $q$ has the following form:

$$
q_{\text {next }}=q_{c u r}+\delta,
$$

where $\delta$ is an appropriate vector to be chosen so that $J\left(q_{\text {next }}\right)<J\left(q_{\text {cur }}\right)$. Many optimization methods have been developed based on how to choose $\delta$ in $(21)[1,3,5,18]$. Since our optimization problem has high nonlinear dependence upon $q$, a modified Levenberg-Marquardt method[18] will be considered.

Consider the following residual vector.

$$
R(q)=\left[R_{1}(q), R_{2}(q), \cdots, R_{m}(q)\right]^{T}, \quad R_{i}(q)=Q\left(t_{i} ; q\right)-w_{i},
$$

where $Q\left(t_{i} ; q\right)$ are the estimated quantities obtained by solving equation (11) for each model), $w_{i}$ are the actual measurement data as in (6), and $m$ is the number of measurements. The next step is to find the gradient of the residual vector $R(q)$ with respect to $q$. Let $\nabla_{q} R$ be the gradient of $R$. Then, from equation (22), we have

$$
\begin{aligned}
\nabla_{q} R & =\left[\nabla_{q} R_{1}, \nabla_{q} R_{2}, \cdots, \nabla_{q} R_{m}\right]^{T} \\
& =\left[\nabla_{q} Q\left(t_{1} ; q\right), \nabla_{q} Q\left(t_{2} ; q\right), \cdots, \nabla_{q} Q\left(t_{m} ; q\right)\right]^{T},
\end{aligned}
$$

where each $\nabla_{q} R_{i}=\nabla_{q} Q\left(t_{i} ; q\right)$ is obtained by the central difference method as in (18). Thus, $\nabla_{q} R(q)$ becomes $m \times 2, m \times 4$, and $m \times 2$ matrices for the power, the exponential, and the logarithmic models, respectively.

The next step is to choose the scalar parameter $\lambda>0$ satisfying the condition that the matrix $\left(\nabla_{q} R\right)^{T}\left(\nabla_{q} R\right)+\lambda I$ to be positive definite, where $I$ is the identity matrix. By controlling the parameter $\lambda$, we can find a descent direction $\delta$ in $(21)$ so that $J\left(q_{\text {next }}\right)<J\left(q_{\text {cur }}\right)$ is satisfied. More precisely, the direction $\delta$ in (21) is obtained by solving the following matrix-vector equation:

$$
\left[\left(\nabla_{q} R\right)^{T}\left(\nabla_{q} R\right)+\lambda I\right] \delta=-\left(\nabla_{q} R\right)^{T} R(q) .
$$


Note that for a small $\lambda$, the system (25) is similar to Newton Method, and for a large $\lambda$, it becomes the Steepest Descent Method[22]. In practice, we choose the parameter $\lambda$ as the following way. Start with an initial guess, say, $\lambda=0.001$, solve equation (25) for $\delta$, set $q_{\text {temp }}=q_{c u r}+\delta$, compute $J\left(q_{\text {temp }}\right)$, and compare it with $J\left(q_{\text {cur }}\right)$. If $J\left(q_{\text {temp }}\right)$ is bigger than $J\left(q_{\text {cur }}\right)$, make $\lambda$ bigger, for example, multiply $\lambda$ by 10 , and continue these process until the condition $J\left(q_{\text {temp }}\right)<J\left(q_{\text {cur }}\right)$ is satisfied. Once we find the descent direction, update $\lambda$ by, for example, dividing $\lambda$ by 10 , update $q$ by setting $q_{\text {next }}=q_{c u r}+\delta$, and continue these steps until $J(q)$ reaches the minimum. Since the minimum value of the cost function $J(q)$ is unknown in advance, the following stopping criterion:

$$
\left|J\left(q_{\text {cur }}\right)-J\left(q_{\text {next }}\right)\right| \leq T o l,
$$

or, equivalently,

$$
\left|q_{\text {next }}-q_{\text {cur }}\right|=\sum_{i=1}^{l}\left|q_{\text {next }}(i)-q_{\text {cur }}(i)\right| \leq \text { Tol }
$$

can be used in practice. Here, Tol is the minimum tolerance value for the convergence of the parameter vectors.

We now summarize our discussion above as the following algorithm, the Central Difference Levenberg-Marquardt Method(CDLMM). The stopping criterion (27) is used for the following algorithm.

\section{Algorithm 2.1(CDLMM)}

0. Input: The first order degradation rate constant $k$ in (1), the number of measurements $m$, and the data set $w=\left[w_{1}, w_{2}, \cdots, w_{m}\right]^{T}$ measured at times $t_{1}, \cdots, t_{m}$ in (6).

1. Choose the maximum iteration number imax, the tolerance Tol for (26) or (27), the time step $\Delta t$ for (11), and the sufficiently small numbers $\Delta q_{i}, i=1,2, \cdots, l$ for (18).

2. Choose an initial parameter $q:=q_{0}$ in (16).

3. Do Steps 4-9 until the stopping criterion (26) or (27) is satisfied.

4. Choose an initial scalar parameter $\lambda:=\lambda_{0}$ in (25).

5. For $i=1,2, \cdots, m$, do Steps 5.1 - 5.3.

5.1. Find $Q\left(t_{i} ; q\right)$ by using the numerical integration formula (11).

5.2. Set the residual $R_{i}=Q\left(t_{i} ; q\right)-w_{i}$.

5.3. Find $\frac{\partial Q\left(t_{i} ; q\right)}{\partial q_{i}}$ by $(18)-(20)$.

6. Compute the cost $J_{\text {cur }}:=\sum_{i=1}^{m}\left(R_{i}\right)^{2}$.

7. Set $J_{\text {next }}=J_{\text {cur }}$ and the iteration counting number $i c=0$. 
8. While $J_{\text {next }}>J_{\text {cur }}$ and $i c<i \max$, do Steps 8.1-8.7.

8.1. Form $\nabla_{q} R$ by equation (23)-(24).

8.2. Find the direction vector $\delta$ from equation (25).

8.3. Let $q=q+\delta$.

8.4. Do Step 5.

8.5. Compute $J_{\text {next }}=\sum_{i=1}^{m}\left(R_{i}\right)^{2}$.

8.6. if $J_{\text {next }}>J_{\text {cur }}$, let $\lambda=\lambda * 10$, else let $\lambda=\lambda * 0.1$.

8.7. Set $i c=i c+1$.

9. Set $q_{\text {next }}=q+\delta$.

3.2.4. Error analysis. To find the most appropriate inflow model among the three given models, the sum of squares of absolutely errors(SSA) and the sum of squares of relative errors(SSR) can be used for each model. The SSA and the SSR are defined by the following.

$$
S S A=\sum_{i=2}^{m}\left(Q_{m e}\left(t_{i} ; q\right)-w_{i}\right)^{2} \quad \text { and } \quad S S R=\sum_{i=2}^{m}\left(\frac{Q_{m e}\left(t_{i} ; q\right)-w_{i}}{w_{i}}\right)^{2},
$$

where $m$ is the number of measurements. Note that the summations in the SSA and the SSR start from $i=2$ due to the fact that $Q_{m e}(0 ; q)=$ $w_{0}=0$.

\section{Numerical results}

The decomposition rate constant $k$ in equation (1) and the measured Clarithromycin remained data(\%) for $k$ are shown in Figure 1. In Figure 1 , the the measured data are shown as circles and the fitted function $100 \exp (-k t)$ with the estimated decomposition rate $k=0.4681$ is shown as a solid line.

To determine the best model among the three proposed inflow models, we used the measured Clarithromycin data(\%) released with $\mathrm{pH} 1.2$. Also, we performed several numerical simulations using the several noise added data with the maximum relative random noise level of $\pm 2 \%$. The measured data and one of typical noise added data are shown in Table 1.

For the numerical estimation of the parameters appeared in the three models, the decomposition rate $k=0.4681$, the small numbers $\epsilon=0.001$ and $\delta=0.001$ for approximating the gradients of the cost function $J$ 


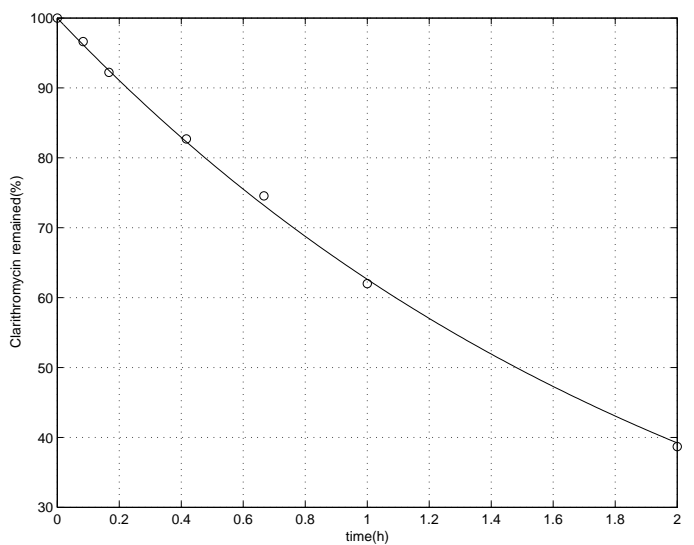

Figure 1. Measured data(o) and $100 \exp (-0.4681 t)$.

TABLE 1. Clarithromycin measured data

\begin{tabular}{|c|c|c|}
\hline time $(\mathrm{h})$ & measured data(\%) & data with $\max \pm 2 \%$ noise \\
\hline 0.0000 & 0.0000 & 0.0000 \\
\hline 1.0000 & 27.8524 & 28.1482 \\
\hline 2.0000 & 27.2115 & 27.5328 \\
\hline 3.0000 & 23.6408 & 23.3447 \\
\hline 4.0000 & 20.4887 & 20.4803 \\
\hline 5.0000 & 16.5910 & 16.5549 \\
\hline 6.0000 & 13.5043 & 13.5833 \\
\hline
\end{tabular}

in (8), the maximum number of iterations $i \max =100$, the time step $\Delta t=0.001$, and the convergence tolerance $T o l=10^{-6}$ were chosen. The stopping criterion (27) was chosen to see the convergence of the parameters to be estimated. For all the models, the numbers of iterations for the convergence of the parameters $q$ were less than 10 for a wide range of initial guesses, and their computational costs were only a few seconds on a PC with the Pentium 4 processor. The estimated parameters, their SSA and SSR for the proposed three models with the measured data and for the noise added data in Table 1 are shown in Tables 2 and 3.

From Table 2, one can observe that, for the measured data, the SSA and the SSR for the exponential inflow model are reduced approximately 
An Appropriate Inflow Model for Simultaneous Dissolution and Degradation 121

TABLE 2. Estimated parameters, SSA, SSR for measured data.

\begin{tabular}{|c|c|c|c|}
\hline Model & Estimated parameter & SSA & SSR \\
\hline Power & $a=42.4389, n=0.3488$ & 1.8393 & $6.5762 \times 10^{-3}$ \\
\hline Exponential & $a=23.6733, b=3.0677$ & 0.1505 & $3.3049 \times 10^{-4}$ \\
& $c=63.1925, d=0.2660$ & & \\
\hline Logarithmic & $a=23.1652, b=0.2604$ & 0.9527 & $1.7602 \times 10^{-3}$ \\
\hline
\end{tabular}

TABLE 3. Estimated parameters, SSA, SSR for data with maximum $\pm 2 \%$ noise.

\begin{tabular}{|c|c|c|c|}
\hline Model & Estimated parameter & SSA & SSR \\
\hline Power & $a=43.0537, n=0.3432$ & 1.6155 & $5.0905 \times 10^{-3}$ \\
\hline Exponential & $a=26.6801, b=2.4530$ & 0.4070 & $8.6333 \times 10^{-4}$ \\
& $c=62.9836, d=0.2329$ & & \\
\hline Logarithmic & $a=22.8511, b=0.2470$ & 1.2257 & $2.5293 \times 10^{-3}$ \\
\hline
\end{tabular}

by $92 \%$ and $95 \%$ compared with those for the power model, and by $84 \%$ and $81 \%$ to the logarithmic model, respectively. For the noise added data, the corresponding reduction rates of the SSA and the SSR for the exponential model were $75 \%$ and $83 \%$ to the power model, and $67 \%$ and $66 \%$ for the logarithmic model, respectively. The numerical simulation results for several other random noised data sets showed that the reduction rates of the exponential model to the power and the logarithmic models were approximately $70-90 \%, 60-80 \%$ for the SSA and $80-90 \%$, $60-80 \%$ for the SSR, respectively. Therefore, based on the numerical simulation results, we can conclude that the exponential inflow model is superior to the power and the logarithmic models. The estimation results for the exponential inflow model are shown for the original measured data in Figure 2 and for the noise added data in Figure 3.

\section{Concluding remarks}

Based on the Clarithromycin measured data released at $\mathrm{pH} 1.2$, we have considered three inflow models: the power, the exponential, and 


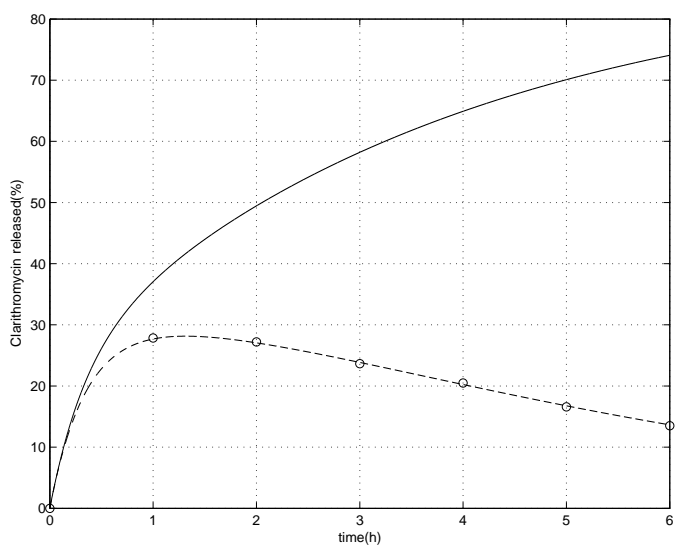

Figure 2. Exponential model: inflow $Q_{i n}(t)(-)$, measured data(o), estimated $Q_{m e}(t)(--)$.

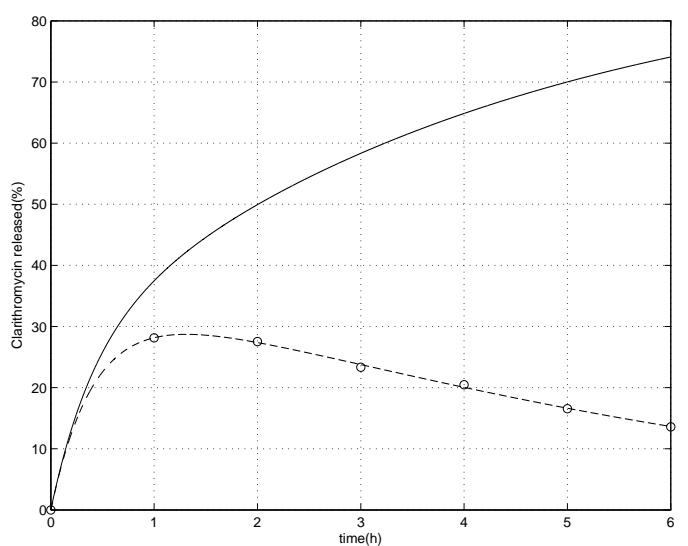

FiguRE 3. Exponential model(with noise): inflow $Q_{i n}(t)(-)$, noised data(o), estimated $Q_{m e}(t)(--)$.

the logarithmic models. The parameters appeared in each model were estimated by the parameter estimation techniques. From the numerical simulations and the error analysis for the measured data and the random noise added data, we can conclude that the exponential model is the best among the three proposed inflow models. The numerical parameter estimation techniques described in this paper can be applied, particularly, to the problems for which the closed form of analytic solution for the governing equations are unknown. Those problems often involve 
An Appropriate Inflow Model for Simultaneous Dissolution and Degradation 123

the nonlinear ordinary differential equations and the partial differential equations.

\section{References}

[1] Ahn, J., Cho, C-K., Kang, S., and Kwon, Y., An efficient parameter estimation technique for a solute transport equation in Porous Media, LNCS 3045, 847-856, Springer-Verlag, 2004.

[2] Allen III, M. B. and Isaacson, E. L., Numerical Analysis for Applied Science, John Wiley \& Sons, 1998.

[3] Banks, H. T. and Kunish, K., Estimation Technique for Distributed Parameter Systems. Birkhauser, Boston, 1989.

[4] Bonzani, I. and Mussone, L., From experiments to hydrodynamic traffic flow models: I-modelling and parameter identification, Math. Comp. Modelling, 37, 1435-1442, 2003

[5] Cho, C-K., Kang, S., and Kwon, Y., Numerical estimation of diffusivity in a nonhysteretic infiltration problem, Comput. Math. Appl., 52, 1511-1528, 2006.

[6] Cho, C-K., Kang, S., and Kwon, Y., Estimation of soil water distribution: Identifiability and observation design, Comput. Math. Appl., 34(2), 105-120, 1997.

[7] Chun, M.-K., Sah, H. and Choi, H.-K., Preparation of mucoadhesive microspheres containing antimicrobial agents for eradication of $\mathrm{H}$. pylori, Int. J. Pharm., 297, 172-179, 2005.

[8] Crison, J.R., Weiner, N.D. and Amidon, G.L., Dissolution media for in vitro testing of water-insoluble drugs: effect of surfactant purity and electrolyte on in vitro dissolution of carbamazepine in aqueous solutions of sodium lauryl sulfate, J. Pharm. Sci., 86, 384-388, 1997.

[9] Erah, P.O., Goddard, A.F., Barrett, D.A., Shaw, P.N. and Spiller, R.C., The stability of amoxicillin, clarithromycin and metronidazole in gastric juice: relevance to the treatment of Helicobacter pylori infection, J. Antimicrob. Chemother., 39, 5-12, 1997.

[10] Farinha, A., Bica, A., Martins, J.M. and Pais, J.P., Dissolution of omeprazole from delayed-release solid oral dosage forms, Drug Dev. Ind. Pharm., 26, 785-790, 2000 .

[11] Gharbo, S.A., Cognion, M.M. and Williamson, M.J., Modified dissolution method for rifampin, Drug Dev. Ind. Pharm., 15, 331-335, 1989.

[12] Irwin, W.J., Li Wan Po, A. and Stephens, J.S., Noxythiolin-high performance liquid chromatographic assay and stability, J. Clin. Hosp. Pharm., 9, 41-51, 1984.

[13] Jumaa, M., Chimilio, L., Chinnaswamy, S., Silchenko, S. and Stella, V.J., Degradation of NSC-281612 (4-[bis[2-[methylsulfonyl]-oxy]ethyl]amino)-2methyl-benzaldehyde), an experimental antineoplastic agent: effect of $\mathrm{pH}$, solvent composition, (SBE)7m--CD, and HP--CD on stability, J. Pharm. Sci., 93, 532-539, 2004.

[14] Khamis, E.F., Abdel-Hamid, M., Hassan, E.M., Eshra, A. and Elsayed, M.A., A stability-indicating first-derivative spectrophotometric assay of acetazolamide and its use in dissolution and kinetic studies, J. Clin. Pharm. Ther., 18, 97-101, 1993. 
[15] Lipka, E. and Amidon, G.L., Setting bioequivalence requirements for drug development based on preclinical data: optimizing oral drug delivery systems, J. Control. Release, 62, 41-49, 1999.

[16] Mordi, M.N., Pelta, M.D., Boote, V., Morris, G.A. and Barber, J., Acidcatalyzed degradation of clarithromycin and erythromycin B: A comparative study using NMR spectroscopy, J. Med. Chem., 43, 467-474, 2000.

[17] Nilssen, L. and Tai, X., Parameter estimation with the augmented Lagrangian method for a parabolic equation, J. Optim. Theory and Appl., 124, 435-453, 2005.

[18] Press, W., Teukolsky, S., Vetterling, W., and Flannery, B., Numerical Recipes in C: The Art of Scientific Computing, Cambridge Univ. Press, New York, 1992.

[19] Quarteroni, A. and Valli, A., Numerical Approximation of Partial Differential Equations, Springer-Verlag, Berlin Heidelberg, 1994.

[20] Shah, V.P. and Ogger, K.E., Comparison of ultraviolet and liquid chromatographic methods for dissolution testing of sodium phenytoin capsules, J. Pharm. Sci., 75, 1113-1115, 1986.

[21] Sonobe, T., Hasumi, S. Yoshino, T., Kobayashi, Y., Kawata, H. and Nagai, T., Digoxin degradation in acidic dissolution medium, J. Pharm. Sci., 69, 410-413, 1980.

[22] Stoer, J. and Bulirsch, R., Introduction to Numerical Analysis, Springer-Verlag, New York, 2002.

[23] Tapia-Albarran, M. and Villafuerte-Robles, L., Assay of amoxicillin sustained release from matrix tablets containing different proporties of Carbopol 971P NF, Int. J. Pharm., 273, 121-127, 2004.

\section{Ju-Hyun Lee}

Department of Mathematics, Chosun University, Gwangju, 501-759, South Korea.

E-mail : jh0911@hanmail.com

Sungkwon Kang (Corresponding author)

Department of Mathematics, Chosun University, Gwangju, 501-759, South Korea.

E-mail : sgkang@chosun.ac.kr.

\section{Hoo-Kyun Choi}

College of Pharmacy, Chosun University,

Gwangju, 501-759, South Korea.

E-mail : hgchoi@chosun.ac.kr 